\title{
Energy-Efficient UAV Relaying Communications to Serve Ground Nodes
}

\author{
Shakil Ahmed, Student Member, IEEE, Mostafa Zaman Chowdhury Senior Member, IEEE, Yeong Min \\ Jang, Member, IEEE
}

\begin{abstract}
This letter studies the energy-efficient unmanned aerial vehicle (UAV) communications to support ground nodes (GNs). The system considers the UAV working as a relay while there is a base station (BS) on the ground. We analyze the UAV energy consumption model to design the energy-efficient UAV trajectory path. We formulate the energy-efficient UAV relaying communication, which considers both throughput and UAV propulsion energy consumption. We optimize joint transmit power of UAV and BS; UAV trajectory, acceleration, and flying speed to maximize the energy-efficient $U A V$ relaying problem. We also introduce a constraint named as information causality constraint (ICC). The main idea of ICC is to guarantee that the UAV receives information from $B S$ in any time slot and forward the only received information to GNs in remaining time slots. The formulated energy-efficiency maximization problem is not convex. Thus, we solve it sub-optimally using the iterative method. Finally, we present the simulation results to validate the efficacy of the proposed algorithm.
\end{abstract}

Index Terms - UAV relay, energy-efficiency, information causality constraint, sub-optimal.

\section{INTRODUCTION}

$\mathbf{R}$ ECENTLY, unmanned aerial vehicles (UAVs) have become an emerging paradigm to support the fifth generation and beyond high-speed wireless communication networks. UAVs can be deployed quickly and cost-effectively as a mobile relay for enlarging coverage and up-gradation of network connectivity. Besides these substantial applications, the UAV communication system encounters many new challenges, particularly how efficiently and optimally it can use onboard energy because UAVs are fundamentally limited by fixed energy supply. The energy-efficiency of the UAV is the total information bits, which are transmitted to the ground nodes (GNs) during the UAV flight time normalized by the total UAV propulsion energy consumption during that time [1]. Moreover, the UAV relay is required to make sure that it forwards the received data from the base station (BS) to the GNs. This constraint is called information causality constraint (ICC).

Researchers have considered various approaches to design energy-efficient UAV networks. Unfortunately, little research has been performed to guarantee the received information is only from BS, while the energy-efficient UAV relaying communication is designed. For example, authors in [1] proposed an algorithm to improve the physical layer security using UAV working as the relay. However, they did not investigate the energy-efficient scenario. On the other hand, authors in

S. Ahmed is with the Department of Electrical and Computer Engineering, The University of Arizona, AZ, USA, (email: shakilahmed@ieee.org).

M. Z. Chowdhury is with the Department of Electrical and Electronic Engineering, Khulna University of Engineering \& Technology, Khulna, Bangladesh, (email: mzceee@ieee.org).

Y. M. Jang is with the Department of Electronics Engineering, Kookmin University, Seoul, Korea, (email: yjang@kookmin.ac.kr).
[2] studied UAV optimal trajectory path to design energyefficient UAV communication considering single GN in the system. Their proposed model considers that the UAV works as an aerial BS. However, they did not consider remote UAVGNs communication, resulting in inefficient UAV relaying communication. Robust resource allocation via joint transmit power of UAV and BS; UAV trajectory location optimization in the presence of uncertain eavesdroppers is proposed in [3]. Though ICC is considered in [3] to support the remote GNs, the model does not consider the energy-efficient UAV relaying communication.

In this letter, we design energy-efficient UAV relaying networks, which jointly optimizes the UAV and BS transmit power; the UAV trajectory path, acceleration, and speed. We propose an iterative algorithm to design energy-efficient UAV relaying communication. To make sure the GNs receive information from the BS via the UAV, we apply ICC in the maximization problem. We also study the UAV propulsion energy consumption model to design energy-efficient UAV relaying communication. We solve the maximization energyefficient problem considering both throughput and UAV energy consumption. We solve the maximization problem suboptimally until it converges. We validate the improved performance of the proposed algorithm via simulation results.

\section{SyStem ModeL}

We consider a wireless communication system, having one BS and one UAV to serve multiple static GNs, for the outdoor environment in a rural area with few obstacles. The set of GNs is defined as $\mathcal{U}$, where $\mathcal{U}=\{1,2,3, \ldots, U\}$. $U$ is the total number of GNs. In the proposed system, the BS establishes a link to the UAV, and UAV establishes the links to the GNs. UAV flies at a fixed altitude such that it can avoid all tall obstacles. The UAV knows the locations of the GNs and the BS. We consider the frequency division multiple access (FDMA), halfduplex, and decode-and-forward (DF) relaying strategies in this letter. The system also considers no direct communication links between the BS to the GNs due to the tall obstacles. We assume that the effects of shadowing and multi-path are negligible.

The UAV serves the GNs in $0 \leq t \leq T$ time horizon due to its limited resource, where $T$ is the UAV flight time. However, the UAV flies a sufficiently long time so that it can guarantee a better quality of service. Without the loss of generality, our proposed system fits the two-dimensional coordinate system for the BS, the UAV, and the GNs. We define the locations of the BS, and the UAV as $\left(x_{b}, y_{b}\right)$ and $(x(t), y(t))$, respectively. The fixed location of GN $u$, where $u \in U$, is $\left(x_{u}, y_{u}\right)$. 
1) Data rate calculation: The UAV flight time is continuous, which is discretized in this letter to avoid complexity. The continuous-time $T$ is divided into $N$ number of the equal, static, and small-time slots, $\rho$, where $\rho=\frac{T}{N}$. The BS to the UAV channel gain can be expressed as $c_{b}[n]=$ $\frac{\beta_{0}}{\left(x[n]-x_{b}\right)^{2}+\left(y[n]-y_{b}\right)^{2}+H^{2}} . \beta_{0}$ defines the channel power gain at reference distance $d_{0}=1 \mathrm{~m}$. $H$ is the altitude. The data rate between the $\mathrm{BS}$ and the UAV can be expressed as $r_{b}[n]=\log _{2}\left(1+\gamma p_{b}[n] c_{b}[n]\right), n=1,2, \ldots, N-1$, where $\gamma=\frac{\beta_{0}}{\sigma^{2}} . \sigma^{2}$ defines the Additive White Gaussian Noise (AWGN) power at the receiver. $p_{b}[n]$ is the $\mathrm{BS}$ transmit power in $n$ time slot. Similarly, the channel gain between the UAV and GN $u$ is $c_{u}[n]=\frac{\beta_{0}}{\left(x[n]-x_{u}\right)^{2}+\left(y[n]-y_{u}\right)^{2}+H^{2}}$. The data rate between the UAV and the GN $u$ can be expressed as $r_{u}[n]=\log _{2}\left(1+\gamma p_{u}[n] c_{u}[n]\right), n=2,3, \ldots, N$, where $p_{u}[n] \in \mathbb{R}_{+}$defines the UAV transmit power in $n$ time slot. The BS sends information to the UAV in any time slot. Then the UAV forwards the received information to the GN $u$ during remaining time slots. By applying ICC [1], it can be expressed as follows:

$$
\sum_{i=1}^{n-1} r_{b}[i] \geq \sum_{i=2}^{n} r_{u}[i], n=2,3, \ldots, N .
$$

From (1), the BS does not transmit information to the UAV at the last time slot, i.e., $r_{b}[N]=0$. Moreover, the UAV does not transmit information at the first time slot as the BS might send information to the UAV at that time, i.e., $r_{u}[1]=0$. It can be written as $r_{u}[1]=r_{b}[N]=0$. The initial and the final UAV locations are $(x[2], y[2])$ and $(x[N], y[N])$, respectively.

2) UAV energy consumption: The propulsion energy consumption is defined as the amount of energy consumed by UAV during flight time [4]. Energy consumption due to the networking to GNs is negligible compared the propulsion energy consumption. UAV energy consumption for GN $u$ can be expressed as:

$$
\delta_{u}[n]=\left(\alpha\left\|v_{u}[n]\right\|^{3}+\frac{\beta}{\left\|v_{u}[n]\right\|}+\frac{\beta\left\|a_{u}[n]\right\|^{2}}{g^{2}\left\|v_{u}[n]\right\|}\right)+\frac{\Delta k}{\rho} .
$$

where $\alpha$ and $\beta$ are constants, depend on UAV weight, wing area, air density, etc. $\Delta k$ is the kinetic energy and expressed as $\frac{1}{2} m\left(\left\|v_{n}[N]\right\|^{2}-\left\|v_{n}[0]\right\|^{2}\right)$, where $m$ is the mass of UAV. $v_{u}[n]$ is the UAV speed. $g$ is gravitational constant and $a_{u}[n]$ is the UAV acceleration.

\section{OPTIMAL ENERGY-EFFICIENT UAV RELAY}

The energy-efficiency maximization problem is:

$$
\begin{aligned}
& \max _{x[n], y[n], p_{b}[n], p_{u}[n], v_{u}[n], a_{u}[n]} \frac{\sum_{u=1}^{U} \sum_{n=2}^{N} r_{u}[n]}{\sum_{u=1}^{U} \sum_{n=2}^{N} \delta_{u}[n]} \\
& \text { s.t. }\left\|v_{u}[n]\right\| \leq v_{\max }, n=2,3, \ldots, N \text {, } \\
& \left\|v_{u}[n]\right\| \geq v_{\min }, n=2,3, \ldots, N \text {, } \\
& \left\|a_{u}[n]\right\| \leq a_{\max }, n=2,3, \ldots, N \text {, } \\
& (x[n+1]-x[n])+(y[n+1]-y[n])=\rho v_{u}+\frac{1}{2} \rho^{2} a_{u}[n], \\
& 0 \leq p_{b}[n] \leq p_{b}^{\max } \\
& 0 \leq p_{u}[n] \leq p_{u}^{\max },
\end{aligned}
$$

(1). where $r_{u}[n]$ is the UAV-user, $u$ rate and $\delta_{u}[n]$ is defined in (2). The UAV flying speed constraint is defined in (3b) and (3c), while (3d) is the UAV acceleration while flying. UAV mobility constraint [5] is deifned in (3e). Moreover, (3f) and (3g) define the BS and UAV transmit power constraints, where $p_{b}^{\max }$ and $p_{u}^{\max }$ are the maximum BS and UAV power, respectively. However, the optimization problem in (3) is not convex. Thus, we apply a sub-optimal solution approach [6], which can solve (3) iteratively. First, we fix the UAV location and acceleration to solve the transmit power of BS and UAV, and speed. In the second step, we optimize the transmit power of BS and UAV, and speed, using the optimal UAV location and acceleration. We keep repeating the process until it converges.

\section{A. Sub-optimal solution 1}

We first optimize the transmit power of the UAV, $p_{u}[n]$, the $\mathrm{BS}, p_{b}[n]$, and UAV speed, $v_{u}[n]$ for an initial fix UAV position $(x[n], y[n])$ and acceleration, $a_{u}[n]$. Thus, we reformulate (3) by removing the constraints that are related to UAV trajectory location and acceleration. The reformulated maximization problem can be written as:

$$
\begin{aligned}
& \max _{p_{u}[n], p_{b}[n], v_{u}[n]} \frac{\sum_{u=1}^{U} \sum_{n=2}^{N} r_{u}[n]}{\sum_{u=1}^{U} \sum_{n=2}^{N} \delta_{u}[n]} \\
& \text { s.t. }(3 e)-(3 g),(1),(3 b),(3 c) .
\end{aligned}
$$

The standard optimization toolbox cannot solve (4) as it is not a convex problem because of (1), (3b), (3c), and (4). We reformulate ICC in (1) by introducing a variable.

$$
\begin{gathered}
\sum_{i=1}^{n-1} r_{b}[i] \geq \sum_{i=2}^{n} \tau_{u}[i], \\
r_{u}[n] \geq \tau_{u}[n] .
\end{gathered}
$$

where $\tau_{u}[n]$ is newly introduced variable. We can rewrite (4) as follows:

$$
\begin{aligned}
& \max _{p_{u}[n], p_{b}[n], v_{u}[n], \tau_{u}[n]} \frac{\sum_{u=1}^{U} \sum_{n=2}^{N} \tau_{u}[n]}{\sum_{u=1}^{U} \sum_{n=2}^{N} \delta_{u}[n]}, \\
& \text { s.t. }(3 e)-(3 g),(3 b),(3 c),(5),(6) .
\end{aligned}
$$

We replace UAV to the GN $u$ data rate from $r_{u}[n]$ to $\tau_{u}[n]$ in (7). However, (7) with its constraints is not yet soluble due to the non-convex denominator, $\sum_{n=2}^{N} \delta_{u}[n]$ and UAV speed constraint in (3c). We also introduce a slack variable, $q_{u}[n]$ in the denominator of (7). Thus, the reformulated optimization problem is:

$$
\begin{aligned}
& \max _{p_{u}[n], p_{b}[n], v_{u}[n], \tau_{u}[n], q_{u}[n]} \frac{\sum_{u=1}^{U} \sum_{n=2}^{N} \tau_{u}[n]}{\sum_{u=1}^{U} \sum_{n=2}^{N} \delta_{u}^{n e w}[n]}, \\
& \text { s.t. } q[n] \geq v_{\min }, n=2,3, \ldots, N, \\
& \left\|v_{u}[n]\right\|^{2} \leq q_{u}^{2}[n], n=2,3, \ldots, N, \\
& (3 e)-(3 g),(3 b),(5),(6) .
\end{aligned}
$$

where

$$
\delta_{u}^{n e w}[n]=\left(\alpha\left\|v_{u}[n]\right\|^{3}+\frac{\beta}{q_{u}[n]}+\frac{\beta\left\|a_{u}[n]\right\|^{2}}{g^{2} q_{u}[n]}\right)+\frac{\Delta k}{\rho} .
$$


The optimization problem is still a fractional problem, which cannot be solved using the standard optimization toolbox. We apply the classical Dinkelbach method [7]. This process guarantees the convergence and hence, the locally optimal solution is achieved.

$$
\begin{aligned}
& \max _{p_{u}[n], p_{b}[n], v_{u}[n], \tau_{u}[n], q_{u}[n]} \sum_{u=1}^{U} \sum_{n=2}^{N}\left[\tau_{u}[n]-\lambda_{i} \delta_{u}^{n e w}[n]\right], \\
& \text { s.t. }(3 e)-(3 g),(3 b),(5),(6),(9),(10) .
\end{aligned}
$$

where $\lambda_{i}$ is a new numerical value that can be iteratively updated as $\left(\tau_{u}[n] / \delta_{u}^{n e w}[n]\right)$. Now, (12) and its related constraints are convex, which is now soluble using the standard convex optimization software, such as CVX [8].

\section{B. The UAV trajectory design}

Now we determine the UAV trajectory and the UAV acceleration using the solution fo the UAV and the BS transmit power, and the UAV speed from Section III-A. The formulated UAV trajectory optimization problem from (3) is:

$$
\begin{aligned}
& \max _{x[n], y[n], a_{u}[n]} \frac{\sum_{u=1}^{U} \sum_{n=2}^{N} r_{u}[n]}{\sum_{u=1}^{U} \sum_{n=2}^{N} \delta_{u}[n]} \\
& \text { s.t. }(1),(3 d),(3 e) .
\end{aligned}
$$

Here (13) with its related constraints is not a convex problem because of the fractional nature of the objective function and (1). We can tackle the non-convexity of the UAV trajectory design by introducing the slack variables. Thus, it is reformulated as follows:

$$
\begin{aligned}
& \max _{x[n], y[n], a_{u}[n], g[n]} \frac{\sum_{u=1}^{U} \sum_{n=2}^{N} r_{u}^{g}[n]}{\sum_{u=1}^{U} \sum_{n=2}^{N} \delta_{u}[n]} \\
& \text { s.t. }\left(x[n]-x_{u}\right)^{2}+\left(y[n]-y_{u}\right)^{2}+H^{2}-g[n] \leq 0, \\
& (1),(3 d),(3 e) .
\end{aligned}
$$

where $\gamma=\frac{\beta_{0}}{\sigma^{2}}$ and $r_{u}^{g}[n]=\log _{2}\left(1+\frac{\gamma p_{u}[n]}{g[n]}\right) \cdot g[n]$ is the slack variables defined as $g \triangleq[g[1], g[2], g[3], \ldots, g[N]]^{\dagger}$. Now we use (5) - (6) to reformulated (1) as follows:

$$
\begin{gathered}
\sum_{i=1}^{n-1} r_{b}[i] \geq \sum_{i=2}^{n} \tau_{u}[i], \\
r_{u}[n] \geq \tau_{u}[n] .
\end{gathered}
$$

where $\tau_{u}[n]$ is the variable. However, (15) - (16) are not convex problem. Introducing the slack variable, (15) can be reformulated as:

$$
\begin{gathered}
\sum_{i=1}^{n-1} r_{b}^{h}[i] \geq \sum_{i=2}^{n} \tau_{u}[i], \\
\left(x[i]-x_{b}\right)^{2}+\left(y[i]-y_{b}\right)^{2}+H^{2}-h[n] \leq 0 .
\end{gathered}
$$

where $r_{b}^{h}[i]=\log _{2}\left(1+\frac{\gamma p_{b}[i]}{h[i]}\right)$ and $h[n]$ is newly added slack variable. Slack variables, $h[n]$ can be expressed as $h \triangleq$ $[h[1], h[2], h[3], \ldots, h[N]]^{\dagger}$. Now let's reformulate (17) using the first order Taylor series expansion at feasible point $h^{f}[i]$ :

$$
\frac{\gamma p_{b}[i]\left(h^{f}[i]-h[i]\right)}{h^{f}[i]\left(h^{f}[i]+\gamma p_{b}[i]\right) \ln 2}+r_{b}^{h^{f}}[i] \leq r_{b}^{h}[i] .
$$

where $r_{b}^{h^{f}}[i]=\left(1+\frac{\gamma p_{b}[i]}{h^{f}[i]}\right)$. Thus, using (19), (15) can be reformulated as:

$$
\sum_{i=2}^{n} \tau_{u}[i] \leq \sum_{i=1}^{n-1}\left[\frac{\gamma p_{b}[i]\left(h^{f}[i]-h[i]\right)}{h^{f}[i]\left(h^{f}[i]+\gamma p_{b}[i]\right) \ln 2}+r_{b}^{h^{f}}[i]\right] .
$$

Similarly, we address the non-convexity of (16). First, we add a new slack variable $m[n]$ :

$$
\begin{gathered}
r_{u}^{m}[n] \geq \tau_{u}[n], \\
\left(x[n]-x_{u}\right)^{2}+\left(y[n]-y_{u}\right)^{2}+H^{2}-m[n] \leq 0 .
\end{gathered}
$$

where $r_{u}^{m}[n]=\log _{2}\left(1+\frac{\gamma p_{u}[n]}{m[n]}\right)$. Now let's reformulate (21) using the first order Taylor series expansion at a feasible point $m^{f}[n]$.

$$
\frac{\gamma p_{u}[n]\left(m^{f}[n]-m[n]\right)}{m^{f}[n]\left(m^{f}[n]+\gamma p_{u}[n]\right) \ln 2}+r_{u}^{m f}[n] \leq r_{u}^{m}[n],
$$

where $r_{u}^{m f}[n]=\left(1+\frac{\gamma p_{u}[n]}{m^{f}[n]}\right)$. Thus, using (23), (16) can be reformulated as follows:

$$
\tau_{u}[n] \leq\left[\frac{\gamma p_{u}[n]\left(m^{f}[n]-m[n]\right)}{m^{f}[n]\left(m^{f}[n]+\gamma p_{u}[n]\right) \ln 2}+r_{u}^{m f}[n]\right] .
$$

We reformulate (14) as follows:

$$
\begin{aligned}
& \max _{x[n], y[n], \tau_{u}[n], m[n], h[n], a_{u}[n]} \frac{\sum_{u=1}^{U} \sum_{n=2}^{N} \tau_{u}[n]}{\sum_{u=1}^{U} \sum_{n=2}^{N} \delta_{u}[n]}, \\
& \text { s.t. }(3 d),(3 e),(18),(20),(22),(24) .
\end{aligned}
$$

Using the first order Taylor series expansion, (25) is reformulated as follows:

$$
\max _{x[n], y[n], \tau_{u}[n], m[n], h[n], a_{u}[n]} \frac{\sum_{u=1}^{U} \sum_{n=2}^{N}\left[\tau_{u}[n]-s[n]\right]}{\sum_{u=1}^{U} \sum_{n=2}^{N} \delta_{u}[n]},
$$

s.t. $(3 d),(3 e),(18),(20),(22),(24)$.

where $s[n]=\frac{\gamma p_{u}\left(z^{f}[n]-z[n]\right)}{\left(z^{f^{2}}[n]+z^{f} \gamma p_{u}[n]\right) \ln 2}$. However, optimization problem is still a fractional problem, which cannot be solved using the standard optimization toolbox. We apply the classical Dinkelbach method as follows:

$$
\max _{x[n], y[n], \tau_{u}[n], m[n], h[n], a_{u}[n]} \sum_{u=1}^{U} \sum_{n=2}^{N}\left[\tau_{u}[n]-s[n]-\lambda_{i} \delta_{u}[n]\right]
$$

s.t. $(3 d),(3 e),(18),(20),(22),(24)$.

Thus, (27) is a convex problem and shares the same solution with (13), which we can solve using the standard optimization software, such as CVX. We encode the approach in Sections III-A and III-B in an efficient algorithm to solve the 


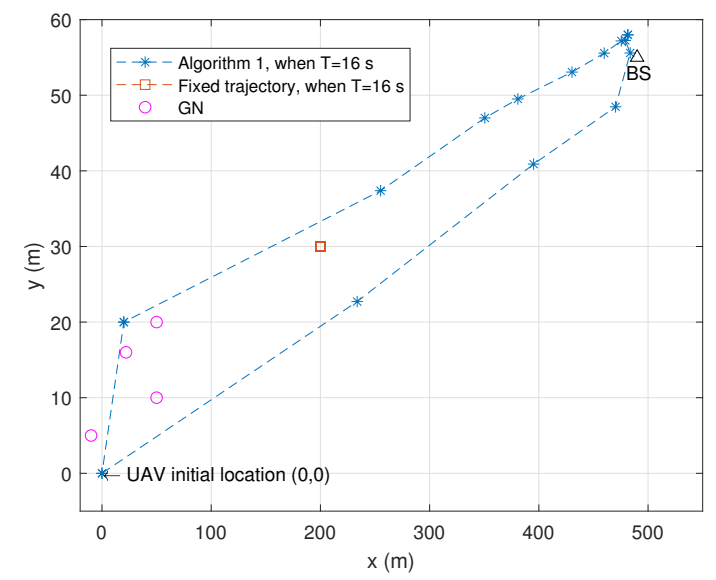

Fig. 1: UAV trajectory design.

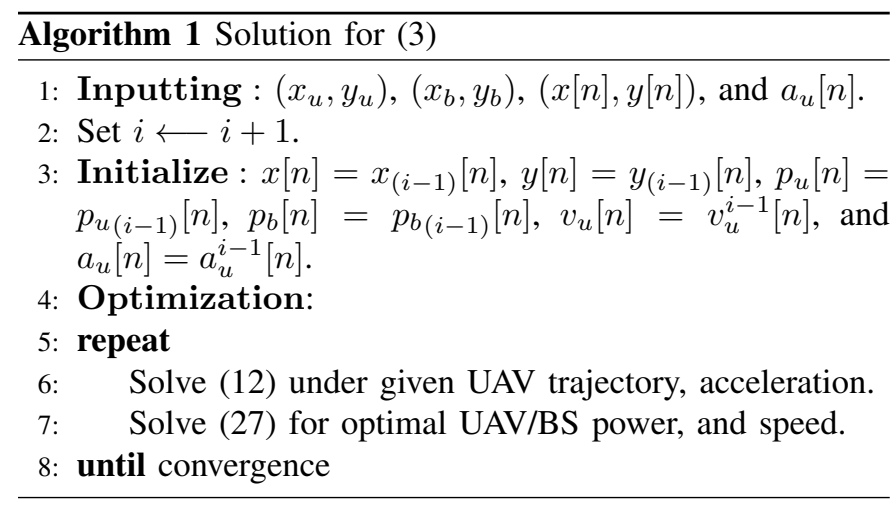

energy-efficiency optimization problem in (3) sub-optimally. We employ successive convex optimization in the algorithm.

The objective value of (3) and its solutions, such as (12) and (27) are increasing over iterations and thus, (3) is finite. Moreover, the solution of the proposed algorithm 1 can be converged to a sub-optimal solution [9] with polynomial time solution and complexity $\mathcal{O}\left[I(4 N+K N)^{3.5}\right]$, where $I$ is the iteration number.

\section{Simulation Results}

Here we present the simulation results of the proposed algorithm and compare it to the UAV having a fixed trajectory. We consider multiple GNs in the system, while the random distribution of the GNs is a Poisson point process. The location of the BS is $(490,55) \mathrm{m} . \gamma=80 \mathrm{~dB}, p_{u}^{\max }=p_{b}^{\max }=10 \mathrm{dBm}$. Fig. 1 shows the UAV path planning and energy-efficiency is illustrated in Fig. 2. Due to ICC, the UAV chooses an optimal path hovering between the BS and the GNs in Fig. 1. This also intuitively makes sense because the UAV is required to hover close to the BS to receive the information and then required to hover close to GNs to send the received information. We present the UAV energy-efficiency versus UAV flight time in Fig 2. Our proposed algorithm has better performance for different UAV altitude. We achieve better performance as ICC makes sure the UAV only forwards the received information from BS at each time slot. Moreover, the proposed algorithm

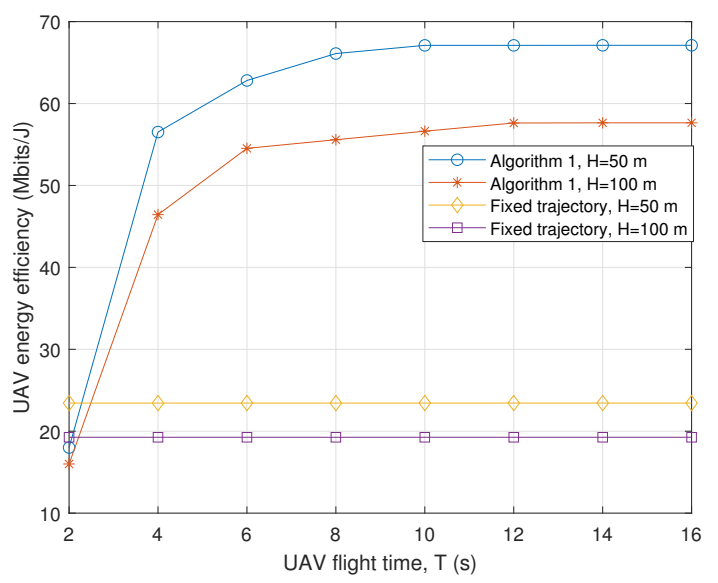

Fig. 2: energy-efficiency.

is energy-efficient even for higher altitudes compared to the other scheme.

\section{Conclusions}

In this letter, we design an energy-efficient UAV relaying communication to support the GNs for a given UAV flight time. We apply the ICC to make sure that the UAV only forwards the received information from the BS to the GNs in remaining time slots. We impose the UAV propulsion energy consumption model to design the energy-efficient UAV relaying communication. We solve the formulated energy-efficiency maximization problem by proposing an iterative algorithm due to the non-convexity, which solves it sub-optimally. The algorithm solves the maximization problem sub-optimally and iteratively until it reaches the optimal global solution. Finally, we show the results to prove the supremacy of the algorithm.

\section{REFERENCES}

[1] L. Xiao, Y. Xu, D. Yang, and Y. Zeng, "Secrecy energy-efficiency maximization for UAV-enabled mobile relaying," IEEE Trans. on Green Commun. and Netw., Oct. 2019.

[2] Q. Wang, Z. Chen, W. Mei, and J. Fang, "Improving physical layer security using UAV-enabled moving relaying," IEEE Comm. Lett., vol. 6, no. 3, pp. 310-313, June 2017.

[3] S. Ahmed, "Robust resource allocation to secure physical layer using UAV-assisted mobile relay communications in 5G technology," MS thesis, College of Engg., Utah State Univ., Utah, Aug. 2019. Accessed on: Aug. 2019. [Online]. Available: https://digitalcommons.usu.edu/etd/ $7575 /$

[4] Y. Zeng and R. Zhang, "Energy-efficient UAV communication with trajectory optimization," IEEE Trans. Wireless Comm., vol. 16, no. 6, pp. 3747-3760, Jun. 2017.

[5] M. Hua, Y. Wang, Z. Zhang, C. Li, Y. Huang and L. Yang, "Powerefficient communication in UAV-aided wireless sensor networks," IEEE Commun. Lett., vol. 22, no. 6, pp. 1264-1267, Jun. 2018.

[6] S. Ahmed and B. Bash, "Average worst-case secrecy rate maximization via UAV and base station resource allocation," in Proc Allerton Conf. on Commun., Cont., and Comput., Monticello, IL, USA, Sept. 2019.

[7] W. Dinkelbach, "On nonlinear fractional programming," Manag. Scien., vol. 13, no. 7, pp. 492-498, Mar. 1967.

[8] M. Grant, S. Boyd, and Y. Ye, "CVX: Matlab software for disciplined convex programming." [Online]. Available: http://www.stanford. edu/boyd/cvx/

[9] Q. Wu, Y. Zeng, and R. Zhang, "Joint trajectory and communication design for multi-UAV enabled wireless networks," IEEE Trans. Wireless Commun., vol. 17, no. 3, pp. 2109-2121, Mar. 2018. 